\title{
Filtros FIR-IFIR Adaptativos sem Efeito de Borda para Cancelamento de Eco de Linha em Sistemas DSL
}

\author{
Eduardo Luiz Ortiz Batista, Orlando José Tobias e Rui Seara
}

\begin{abstract}
Resumo-Este trabalho apresenta um novo algoritmo para o cancelamento de eco em sistemas DSL (digital subscriber line) usando filtros FIR-IFIR adaptativos. A motivação desse algoritmo é usar uma estrutura com remoção de efeito de borda para o filtro IFIR visando eliminar perdas de desempenho oriundas da associação dos filtros FIR-IFIR em aplicações de cancelamento de eco. Dessa forma, um algoritmo com desempenho superior ao de outras abordagens FIR-IFIR encontradas na literatura é obtido. Resultados de simulação são mostrados atestando a efetividade do algoritmo proposto.
\end{abstract}

Palavras-chave-Algoritmo LMS, cancelamento de eco, filtros adaptativos, filtros FIR interpolados.

Abstract-This paper presents a novel algorithm for line echo cancellation in digital subscriber line (DSL) systems using adaptive FIR-IFIR filters. The motivation of this algorithm is to use an IFIR filter with removed boundary effect aiming to eliminate the loss of performance arisen from the FIR-IFIR combination for echo cancellation applications. Thereby, an algorithm with a superior performance as compared with other FIR-IFIR approaches from the literature is obtained. Simulation results are presented attesting the effectiveness of the proposed algorithm.

Keywords - LMS algorithm, echo cancellation, adaptive filters, interpolated FIR filters.

\section{INTRODUÇÃO}

Um problema recorrente em comunicações através de linhas telefônicas é a presença de sinais de eco [1]. Tais sinais podem ser classificados como eco acústico ou eco de linha. $\mathrm{O}$ primeiro tem origem em sistemas viva-voz [2], produzindo um indesejável acoplamento acústico entre o alto-falante e o microfone; enquanto o segundo é devido principalmente ao descasamento de impedâncias na conversão de quatro para dois fios [1]. O problema do eco de linha ganhou maior

Eduardo L. O. Batista e Rui Seara, LINSE - Laboratório de Circuitos e Processamento de Sinais, Depto. de Eng. Elétrica, Universidade Federal de Santa Catarina, Florianópolis, SC, E-mails: \{dudu, seara\}@linse.ufsc.br.

Orlando J. Tobias, LINSE/UFSC e Depto. Eng. Elétrica e Telecom., Universidade Regional de Blumenau, SC, E-mail: tobias.oj@ieee.org.

Este trabalho foi parcialmente financiado pela Coordenação de Aperfeiçoamento de Pessoal de Nível Superior (CAPES) e pelo Conselho Nacional de Desenvolvimento Científico e Tecnológico (CNPq). importância nas últimas décadas com a difusão dos sistemas DSL (digital subscriber line) [3] e a conseqüente transmissão de dados a taxas elevadas pela rede telefônica. Nesse contexto, a utilização de sistemas de cancelamento de eco torna-se indispensável para garantir comunicações confiáveis e de boa qualidade.

As respostas ao impulso de eco encontradas em sistemas DSL são usualmente longas [4], [5]. Assim, o uso de filtros FIR adaptativos para o cancelamento de eco implica um grande número de coeficientes e, conseqüentemente, uma alta complexidade computacional é requerida nesses casos. Então, visando reduzir o custo computacional, abordagens baseadas em estruturas híbridas FIR-IFIR vêm sendo consideradas com sucesso [4], [5]. A proposta é usar um filtro FIR adaptativo para o cancelamento do eco associado à parte inicial da resposta ao impulso e um filtro interpolado FIR adaptativo (IFIR) [6] para o cancelamento da parte final da correspondente resposta. Trata-se de uma estratégia interessante, visto que a resposta ao impulso de eco típica $h(n)$ (encontrada em sistemas DSL) possui uma porção inicial de curta duração com variações rápidas e uma parte final mais longa, variando lentamente, conforme ilustrado na Fig. 1 [4], [5]. Essa característica particular correspondente à parte final da reposta ao impulso implica um maior grau de correlação entre amostras próximas e/ou vizinhas, permitindo uma modelagem eficiente com o uso de um filtro IFIR. Como resultado, as estruturas FIR-IFIR têm obtido desempenho muito bom quando aplicadas ao cancelamento de eco para sistemas DSL [4], [5].

A abordagem FIR-IFIR foi originalmente proposta em [4], sendo que, nesse trabalho, apenas o cancelamento do eco relacionado à parte final da resposta ao impulso de eco, obtido pelo filtro IFIR, foi discutido. No entanto, as dificuldades conseqüentes da associação dos filtros FIR e IFIR para a síntese do cancelador de eco não foram discutidas em [4]. A principal dificuldade está relacionada ao efeito de borda oriundo da operação de convolução entre o interpolador e o filtro esparso que compõem a estrutura de um filtro IFIR [5], [7]. Em [5], uma outra implementação de um cancelador de eco FIR-IFIR é proposta, visando resolver os problemas negligenciados em [4]. Apesar do desempenho muito bom obtido, a abordagem adotada em [5] implica um indesejável aumento de complexidade computacional. Além disso, uma redução na velocidade de convergência do algoritmo adaptativo é também verificada. 


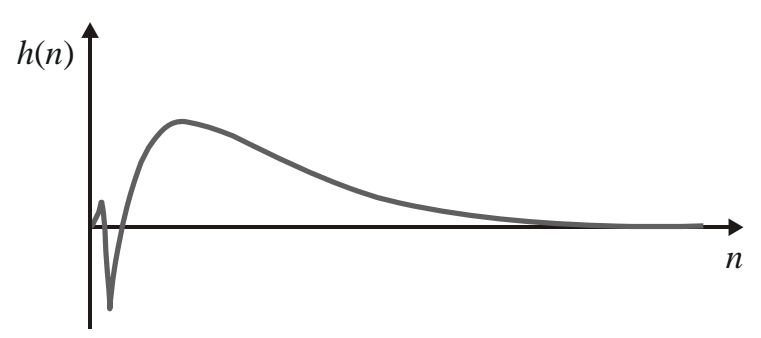

Fig. 1. Resposta ao impulso de eco típica de uma híbrida de sistemas DSL.

Contribuindo nesse contexto, uma nova abordagem FIR-IFIR para o cancelamento de eco em sistemas DSL é aqui proposta. Tal abordagem baseia-se na implementação do filtro IFIR da estrutura FIR-IFIR usando o procedimento de remoção de efeito de borda descrito em [7] e [8]. Assim, torna-se possível mitigar os problemas de implementação do algoritmo FIR-IFIR proposto em [4], sem aumento significativo de carga computacional. Como conseqüência, um algoritmo para o cancelamento de eco em sistemas DSL com baixo custo computacional e com desempenho muito bom é obtido.

Este artigo está organizado como segue. Na Seção II, as abordagens FIR-IFIR para o cancelamento de eco em sistemas DSL são descritas, com destaque para o papel do efeito de borda do filtro IFIR. Na Seção III, a implementação de filtros FIR-IFIR usando o procedimento de remoção do efeito de borda é apresentada e o seu custo computacional é discutido. A Seção IV apresenta resultados de simulação atestando o bom desempenho da abordagem proposta. Finalmente, na Seção V, as conclusões deste trabalho são apresentadas.

\section{ESTRUTURAS FIR-IFIR ADAPTATIVAS PARA CANCELAMENTO DE ECO EM SISTEMAS DSL}

O diagrama de blocos de uma estrutura FIR-IFIR adaptativa é mostrado na Fig. 2. Nessa figura, $\mathbf{w}_{\mathrm{h}}$ representa um filtro FIR, $\mathbf{w}_{\mathrm{t}}$ um filtro IFIR e $z^{-D}$ um atraso de $D$ amostras. A variável $x(n)$ denota o sinal de entrada do filtro e $y(n)$, o sinal de saída.

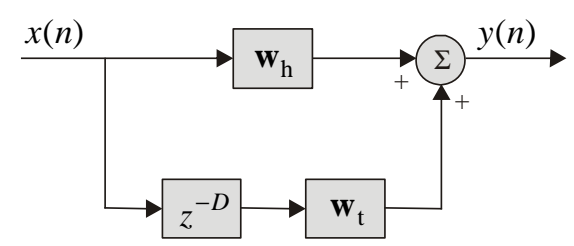

Fig. 2. Diagrama de blocos de uma estrutura FIR-IFIR adaptativa.

Conforme mencionado anteriormente, a estrutura FIR-IFIR foi originalmente apresentada em [4]. Nesse trabalho, apenas o cancelamento do eco relacionado à parte final da resposta ao impulso de eco, realizado pelo filtro IFIR $\mathbf{w}_{t}$, foi tratado. $\mathrm{O}$ filtro IFIR $\mathbf{w}_{\mathrm{t}}$ é composto por um interpolador $\mathbf{g}$ em cascata com um filtro esparso $\mathbf{w}_{\mathrm{ts}}$ [4], [8]. O interpolador $\mathbf{g}$ possui tamanho de memória $M$, representado pelo seguinte vetor:

$$
\mathbf{g}=\left[\begin{array}{llll}
g_{0} & g_{1} & \cdots & g_{M}
\end{array}\right]^{\mathrm{T}} .
$$

O filtro esparso $\mathbf{w}_{\text {ts }}$ tem tamanho da memória $N$, sendo denotado pelo seguinte vetor:

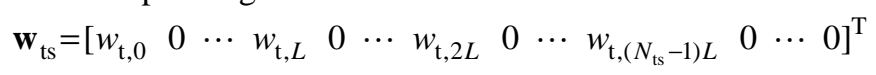

onde $N_{\text {ts }}=[(N-D-1) / L]+1$ é o número de coeficientes diferentes de zero em $\mathbf{w}_{\text {ts }}$ e $L$, o fator de interpolação ou esparsidade [7]. O tamanho de memória do interpolador é geralmente relacionado ao fator de interpolação através da seguinte expressão [7]:

$$
M(L)=2 L-1
$$

$\mathrm{O}$ vetor de coeficientes equivalente do filtro IFIR $\mathbf{w}_{\mathrm{t}}$, correspondente à sua resposta ao impulso, é dado por [7]

$$
\mathbf{w}_{\mathrm{t}}=\mathbf{g} * \mathbf{w}_{\mathrm{ts}} .
$$

Por exemplo, considerando um caso em que $N=7$ e $L=2$, tem-se $M=3, \quad \mathbf{g}=\left[\begin{array}{lll}g_{0} & g_{1} & g_{2}\end{array}\right]^{\mathrm{T}}, \quad \mathbf{w}_{\mathrm{ts}}=\left[\begin{array}{lllll}w_{\mathrm{t}, 0} & 0 & w_{\mathrm{t}, 2} & 0 & w_{\mathrm{t}, 4}\end{array}\right.$ $\left.0 w_{t, 6}\right]^{T}$ e um vetor de coeficientes equivalente dado por

$$
\begin{array}{r}
\mathbf{w}_{\mathrm{t}}=\left[\begin{array}{llll}
g_{0} w_{t, 0} & g_{1} w_{t, 0} & \left(g_{2} w_{t, 0}+g_{0} w_{t, 2}\right) g_{1} w_{t, 2}\left(g_{2} w_{t, 2}+g_{0} w_{t, 4}\right) \\
& g_{1} w_{t, 4}\left(g_{2} w_{t, 4}+g_{0} w_{t, 6}\right) g_{1} w_{t, 6} \quad g_{2} w_{t, 6}
\end{array}\right]^{\mathrm{T}} .
\end{array}
$$

Considerando, agora, o uso de um interpolador linear [5], com $\mathbf{g}=\left[\begin{array}{lll}0,5 & 1 & 0,5\end{array}\right]^{\mathrm{T}}$ em (5), obtém-se [7]

$$
\mathbf{w}_{\mathrm{t}}=\left[\begin{array}{l}
0,5 w_{t, 0} \\
\left.w_{t, 0} \frac{\left(0,5 w_{t, 0}+0,5 w_{t, 2}\right)}{w_{t, 4}\left(0,5 w_{t, 4}+0,5 w_{t, 6}\right)} w_{t, 6} 0,5 w_{t, 6}\right]^{\mathrm{T}}
\end{array}\right.
$$

Agora, comparando (2) com (6), observa-se que [7]: (i) os coeficientes do filtro esparso $\mathbf{w}_{\mathrm{ts}}$ são reproduzidos em $\mathbf{w}_{\mathrm{t}}$; (ii) os coeficientes zerados do filtro esparso $\mathbf{w}_{\text {ts }}$ são recriados em $\mathbf{w}_{t}$ (coeficientes indicados por caixas); e (iii) novos coeficientes (sublinhados) aparecem como conseqüência do efeito de borda. Os coeficientes correspondentes ao efeito de borda do filtro IFIR $\mathbf{w}_{\mathrm{t}}$ têm um impacto bastante negativo no desempenho da estrutura FIR-IFIR. Conforme descrito em [5], a resposta ao impulso de um filtro IFIR apresenta uma indesejável característica em forma de rampa em seu início e em seu final quando interpoladores lineares são considerados. Tal característica fica evidente aplicando em (6) um vetor de coeficientes esparso como, por exemplo, $\mathbf{w}_{\mathrm{ts}}=\left[\begin{array}{lll}0,5 & 0 & 0,38\end{array}\right.$ $\left.\begin{array}{llll}0 & 0,4 & 0 & 0,45\end{array}\right]^{\mathrm{T}}$. Como resultado, obtém-se um vetor cujos valores dos coeficientes estão ilustrados na Fig. 3, com a parte correspondente ao efeito de borda em destaque. A partir dessa figura, observa-se claramente a característica em forma de rampa, apontando para zero no início e no fim do vetor 
resultante [5]. Como conseqüência, a associação do filtro FIR com o filtro IFIR, conforme sugerido em [4], origina uma resposta ao impulso, a qual é ilustrada na Fig. 4. Nessa figura, observa-se uma importante diferença entre a resposta ao impulso a ser modelada (em cinza) e a obtida com os filtros FIR (linha pontilhada) e IFIR (linha tracejada), o que leva a uma significativa degradação de desempenho.

Uma estratégia para mitigar o impacto da distorção causada pelo efeito de borda no desempenho do filtro FIR-IFIR é proposta em [5]. Tal estratégia, ilustrada na Fig. 5, consiste em aumentar o tamanho da memória do filtro FIR e impor uma superposição de parte de sua resposta ao impulso com a parte da resposta do filtro IFIR correspondente ao efeito de borda. Assim, alguns dos coeficientes do filtro FIR são utilizados com o objetivo de compensar a distorção causada pelo efeito de borda do filtro IFIR. Apesar do bom desempenho obtido com a superposição das respostas ao impulso dos filtros FIR e IFIR, um aumento de complexidade computacional é verificado em conseqüência do aumento no tamanho de memória do filtro FIR. Além disso, observa-se, a partir dos resultados apresentados em [5], a necessidade de se aumentar também o tamanho de memória do interpolador de forma a garantir um desempenho satisfatório do algoritmo. Por exemplo, para os casos apresentados em [5] em que o fator de interpolação é $L=4$, um interpolador com 23 coeficientes foi utilizado, enquanto usualmente 7 coeficientes seriam necessários [ver (3)]. Esse aumento do tamanho de memória do interpolador também implica aumento de carga computacional. Adicionalmente, em [5], menciona-se que uma redução na velocidade de convergência do algoritmo adaptativo é verificada em conseqüência da superposição das respostas ao impulso dos filtros FIR e IFIR. Tal redução é amenizada zerando alguns dos coeficientes do filtro FIR na região de sua resposta ao impulso onde ocorre a superposição com a resposta do filtro IFIR [5].

\section{ABORDAGEM FIR-BIFIR}

Nesta seção, uma nova abordagem FIR-IFIR para cancelamento de eco em sistemas DSL é apresentada. A idéia central aqui é implementar o filtro IFIR da estrutura FIR-IFIR usando a implementação IFIR com remoção de efeito de borda (BIFIR) [7], [8]. Tal abordagem, denominada FIR-BIFIR, permite eliminar a distorção causada pelo efeito de borda sem necessidade de superposição de coeficientes dos filtros FIR e IFIR, conforme ilustrado na Fig. 6.

O diagrama de blocos do filtro FIR-BIFIR é idêntico ao do filtro FIR-IFIR mostrado na Fig. 2. O filtro FIR $\mathbf{w}_{\mathrm{h}}$ possui tamanho de memória igual ao atraso de $D$ amostras. Assim, seu vetor de coeficientes é dado por

$$
\mathbf{w}_{\mathrm{h}}=\left[\begin{array}{lllll}
w_{\mathrm{h}, 0} & w_{\mathrm{h}, 1} & w_{\mathrm{h}, 2} & \cdots & w_{\mathrm{h}, D-1}
\end{array}\right]^{\mathrm{T}}
$$

e o vetor de entrada correspondente por

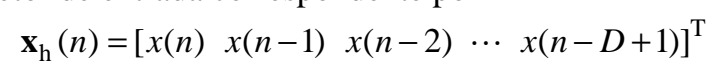

o que resulta na seguinte relação de entrada e saída:

$$
y_{\mathrm{h}}(n)=\mathbf{w}_{\mathrm{h}}^{\mathrm{T}} \mathbf{x}_{\mathrm{h}}(n) .
$$

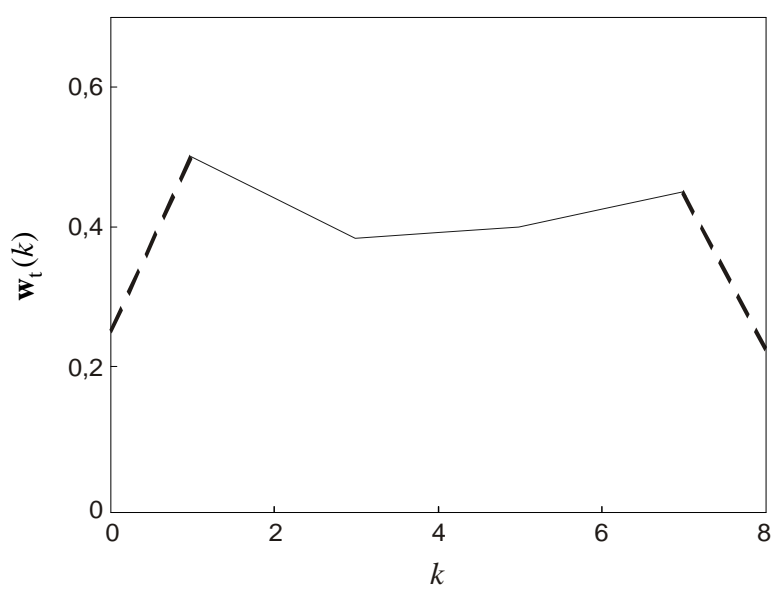

Fig. 3. Resposta ao impulso de um filtro IFIR com as partes correspondentes ao efeito de borda indicadas em linhas tracejadas escuras.

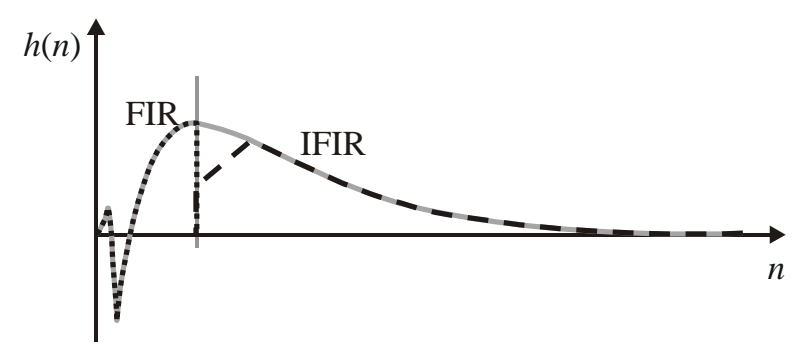

Fig. 4. Resposta ao impulso do filtro FIR-IFIR proposto em [4].

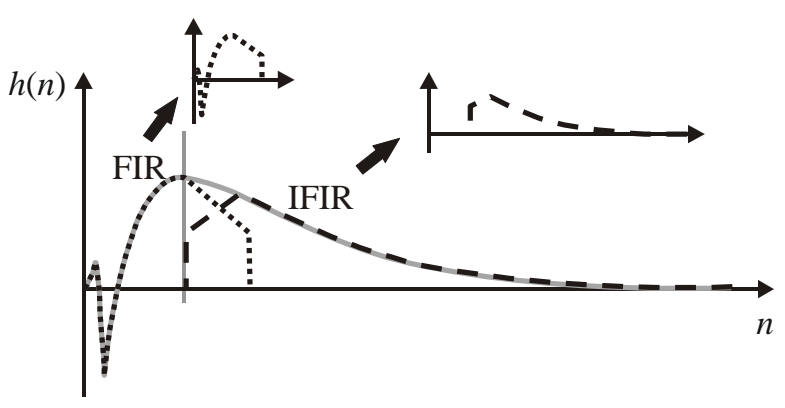

Fig. 5. Resposta ao impulso do filtro FIR-IFIR proposto em [5].

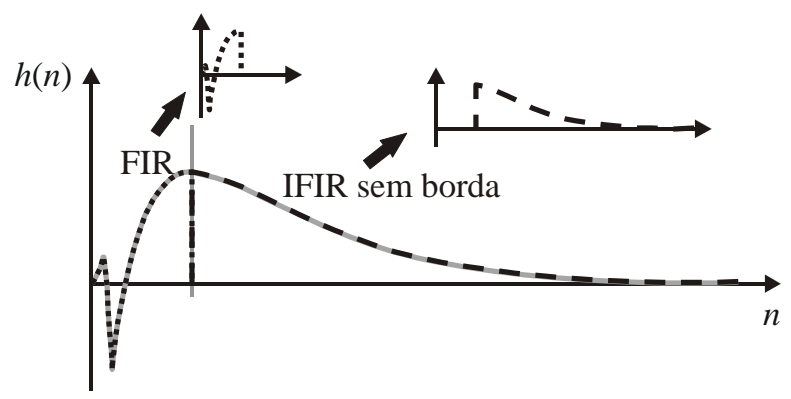

Fig. 6. Resposta ao impulso da abordagem FIR-BIFIR proposta. 
Conforme mencionado anteriormente, a principal diferença entre as estruturas FIR-IFIR e FIR-BIFIR está na forma de implementação do filtro IFIR $\mathbf{w}_{\mathrm{t}}$. No caso da abordagem FIR-BIFIR, $\mathbf{w}_{\mathrm{t}}$ é implementado com a remoção do efeito de borda proposta em [7]. Assim, o vetor de coeficientes esparso é o mesmo da abordagem FIR-IFIR, dado por (2), enquanto o vetor de entrada interpolado do filtro esparso é agora dado por

$$
\tilde{\mathbf{x}}_{\mathrm{t}}^{\prime}(n)=\mathbf{G}^{\mathrm{T}} \mathbf{T}^{\mathrm{T}} \mathbf{x}_{\mathrm{t}}(n) .
$$

Em (10), as matrizes $\mathbf{G}$ de interpolação e $\mathbf{T}$ de transformação [7], e o vetor de entrada $\mathbf{x}_{\mathrm{t}}(n)$ são definidos, respectivamente, como

$$
\begin{aligned}
& \mathbf{G}=\left[\begin{array}{ccccc}
g_{0} & 0 & 0 & \cdots & 0 \\
g_{1} & g_{0} & 0 & \cdots & 0 \\
g_{2} & g_{1} & g_{0} & \cdots & 0 \\
\vdots & \vdots & \vdots & \ddots & \vdots \\
g_{M-1} & g_{M-2} & g_{M-3} & \cdots & g_{0} \\
0 & g_{M-1} & g_{M-2} & \cdots & g_{1} \\
0 & 0 & g_{M-1} & \cdots & g_{2} \\
\vdots & \vdots & \vdots & \ddots & \vdots \\
0 & 0 & 0 & \cdots & g_{M-1}
\end{array}\right]
\end{aligned}
$$

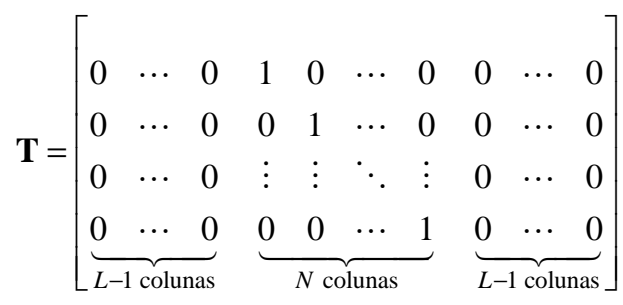

e

$$
\begin{aligned}
& \mathbf{x}_{\mathrm{t}}(n)=[x(n-D) \quad x(n-D-1) \\
& x(n-D-2) \cdots x(n-N+D+1)]^{\mathrm{T}} .
\end{aligned}
$$

Considerando (2) e (10), a relação de entrada e saída do filtro IFIR com efeito de borda removido (BIFIR) pode ser agora escrita como

$$
y_{\mathrm{t}}(n)=\mathbf{w}_{\mathrm{t}}^{\mathrm{T}} \tilde{\mathbf{x}}_{\mathrm{t}}^{\prime}(n)
$$

Apesar da aparente complexidade de cálculo do vetor de entrada $\tilde{\mathbf{x}}_{\mathrm{t}}^{\prime}(n)$ do filtro BIFIR, sua implementação de forma recursiva tem um custo computacional bastante baixo [7]. De maneira geral, a implementação de (10) resulta em um acréscimo de apenas $2 L-2$ operações por amostra em comparação com o cálculo do vetor de entrada interpolado de um filtro IFIR convencional. Como $L$ é geralmente pequeno, o aumento em termos de complexidade computacional é praticamente desprezível. Finalmente, considerando o algoritmo LMS [1] para adaptação dos coeficientes, tem-se

$$
\mathbf{w}_{\mathrm{h}}(n)=\mathbf{w}_{\mathrm{h}}(n-1)+2 \mu_{\mathrm{h}} e(n) \mathbf{x}_{\mathrm{h}}(n)
$$

$$
\mathbf{w}_{\mathrm{t}}(n)=\mathbf{w}_{\mathrm{t}}(n-1)+2 \mu_{\mathrm{t}} e(n) \tilde{\mathbf{x}}_{\mathrm{t}}^{\prime}(n)
$$

onde $\mu_{\mathrm{h}}$ e $\mu_{\mathrm{t}}$ são os passos de adaptação e

$$
e(n)=d(n)-y(n)=d(n)-y_{\mathrm{h}}(n)-y_{\mathrm{t}}(n)
$$

é o sinal de erro com $d(n)$ denotando o sinal de retorno com eco.

\section{Complexidade Computacional}

A complexidade computacional requerida para implementação do filtro FIR-BIFIR adaptado pelo algoritmo LMS está descrita, em termos do número de operações por amostra, na Tabela I. A comparação de carga computacional da abordagem FIR-BIFIR com àquela requerida pela implementação FIR-IFIR de [5] deve, no entanto, ser realizada com certa cautela. Isso se deve ao fato de o filtro FIR-IFIR de [5] apresentar diversos parâmetros que podem ser ajustados, o que leva a diferentes configurações com diferentes cargas computacionais. Com o objetivo de realizar uma comparação consistente, a configuração do filtro FIR-IFIR utilizada para obter os resultados de simulação em [5] é aqui considerada. Assim, tem-se $N=250, L=4$, $D=31$, um filtro FIR com 50 coeficientes e um interpolador com 23 coeficientes. No caso do filtro FIR-BIFIR, a escolha dos parâmetros é mais simples, sendo necessário apenas estabelecer $N=250, \quad L=4$ e $D=31$, o que resulta em um filtro FIR com $D=31$ coeficientes e um interpolador com $M=7$ coeficientes. A Tabela II apresenta uma comparação, para o caso descrito, da carga computacional requerida para o filtro FIR convencional, o filtro FIR-IFIR de [4], o FIR-IFIR de [5] e o FIR-BIFIR proposto. A partir dessa tabela, observa-se que as abordagens FIR-IFIR e a FIR-BIFIR apresentam uma complexidade computacional muito inferior à do filtro FIR adaptativo. Adicionalmente, constata-se que a abordagem FIR-BIFIR apresenta complexidade similar à do FIR-IFIR de [4] e, ainda, uma redução de complexidade de $18,5 \%$ em relação ao filtro FIR-IFIR de [5].

TABELA I

NÚMERO DE OPERAÇÕES POR AMOSTRA REQUERIDO PARA IMPLEMENTAÇÃO DO FILTRO FIR-BIFIR

\begin{tabular}{l|c|c|c|c}
\hline & \multicolumn{2}{|c|}{ Filtro FIR $\mathbf{w}_{\mathrm{h}}$} & \multicolumn{2}{c}{ Filtro BIFIR $\mathbf{w}_{\mathrm{t}}$} \\
\hline & Filtragem & Adaptação & Filtragem & Adaptação \\
\hline Multiplicações & $D$ & $D$ & $M+N_{\text {ts }}+L-1$ & $N_{\text {ts }}$ \\
\hline Somas & $D-1$ & $D$ & $M+N_{\text {ts }}+L-3$ & $N_{\text {ts }}$ \\
\hline
\end{tabular}

TABELA II

COMPARAÇÃO DE COMPLEXIDADE COMPUTACIONAL ENTRE DIFERENTES IMPLEMENTAÇÕES DE FILTROS: FIR, FIR-IFIR E FIR-BIFIR, COM $N=250, L=4$ E $D=31$

\begin{tabular}{l|c|c|c}
\hline & $\begin{array}{c}\text { Operações por } \\
\text { amostra }\end{array}$ & \multicolumn{2}{|c}{$\begin{array}{c}\text { Percentuais } \\
\text { relativos }\end{array}$} \\
\hline FIR convencional & 999 & $\mathbf{1 0 0 \%}$ & \\
\hline FIR-IFIR [4] & 355 & $35,5 \%$ & \\
\hline FIR-IFIR [5] & 443 & $44,3 \%$ & $\mathbf{1 0 0 \%}$ \\
\hline FIR-BIFIR (proposto) & 361 & $36,1 \%$ & $81,5 \%$ \\
\hline
\end{tabular}




\section{REsultados de SimulaçÃo}

Com o objetivo de avaliar o desempenho do filtro FIR-BIFIR em comparação com o das outras implementações FIR-IFIR, nesta seção, são apresentados alguns resultados de simulação. Os diferentes filtros são aplicados a um problema de cancelamento de eco cuja resposta ao impulso, apresentada na Fig. 7, possui características similares às respostas consideradas em [5]. Os tamanhos de memória, fatores de esparsidade e valores de atraso considerados são os mesmos utilizados para a comparação de complexidade descrita na seção anterior. O desempenho é avaliado em termos da medida ERLE (echo return loss enhancement), definida como [5]

$$
E R L E=10 \log _{10} \frac{E\left[d^{2}(n)\right]}{E\left[e^{2}(n)\right]}
$$

onde $d(n)$ é o sinal de retorno com eco e $e(n)$, o sinal com o eco cancelado. As curvas de ERLE foram obtidas por simulação de Monte Carlo (média de 100 realizações). De forma similar a [5], o sinal de entrada é um sinal codificado em 16-PAM com distribuição uniforme de probabilidade entre seus diferentes níveis.

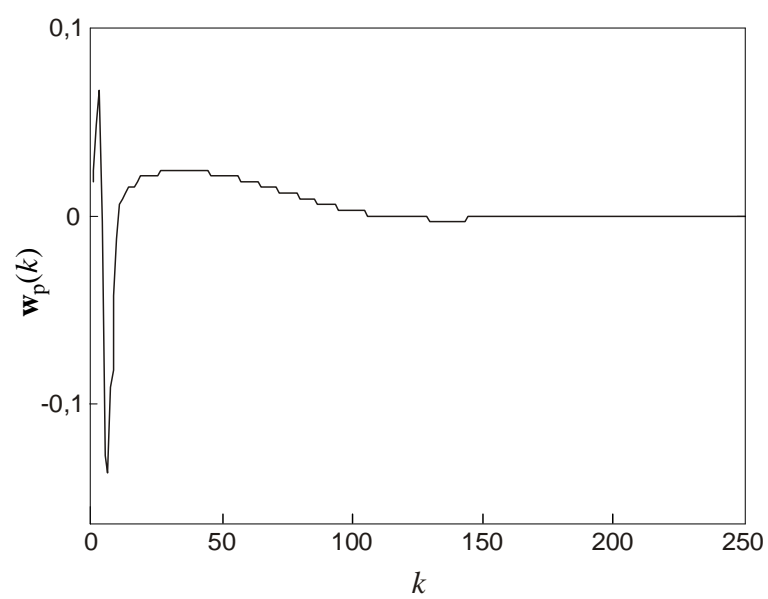

Fig. 7. Resposta ao impulso de eco de um sistema de comunicação DSL.

Os resultados de simulação obtidos são apresentados na Fig. 8. A Fig. 8(a) mostra os resultados das três implementações avaliadas utilizando como passo de adaptação para os algoritmos a mesma estratégia descrita em [5]. Dessa forma, o intervalo total de adaptação de 12.000 amostras é dividido em cinco estágios e, a cada estágio, o passo de adaptação é reduzido pela metade. O valor inicial do passo de adaptação é igual a um valor crítico $\mu_{\text {crit }}$ que garante a estabilidade do algoritmo (obtido experimentalmente). Nota-se, a partir dos resultados da Fig. 8(a), que o algoritmo proposto (FIR-BIFIR) apresenta um desempenho superior ao das demais implementações FIR-IFIR. A Fig. 8(b) apresenta os resultados de simulação considerando o uso do próprio $\mu_{\text {crit }}$ como passo de adaptação, mantendo o seu valor fixo ao longo de todo o processo adaptativo. Nesse caso, observa-se uma melhoria no desempenho do filtro FIR-IFIR de [5] em comparação com o caso anterior usando passo variável, sendo ainda FIR-BIFIR o filtro que mostra o melhor desempenho. Uma terceira estratégia de escolha do passo de adaptação é o uso de um valor do passo para o filtro FIR e um outro valor diferente para o filtro interpolado das estruturas FIR-IFIR e FIR-BIFIR. A Fig. 8(c) apresenta os resultados de simulação, verificando-se também, neste último caso, um melhor desempenho da abordagem proposta. Assim, observase que, além de proporcionar um custo computacional similar ao do algoritmo FIR-IFIR descrito em [4] e menor do que aquele da abordagem FIR-IFIR discutida em [5], o algoritmo proposto apresenta desempenho superior ao dos demais algoritmos FIR-IFIR considerados tanto para o valor máximo de ERLE quanto para a velocidade de convergência.

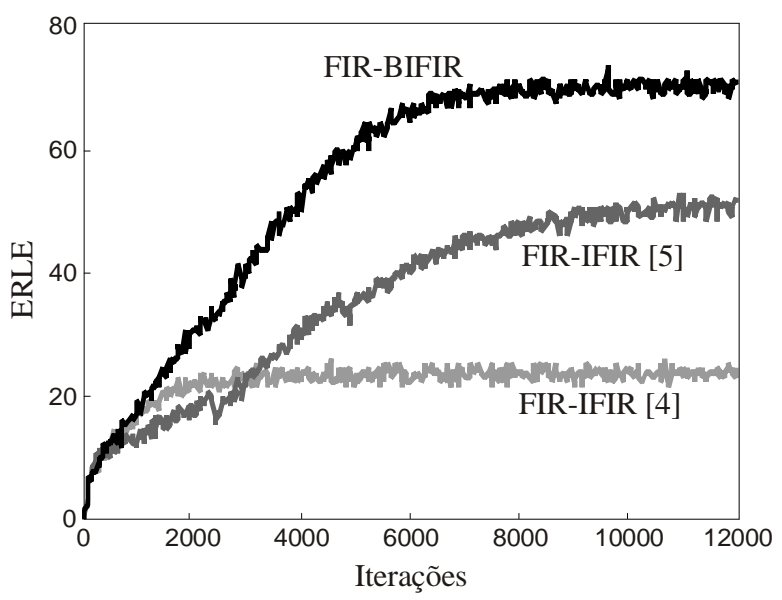

(a)

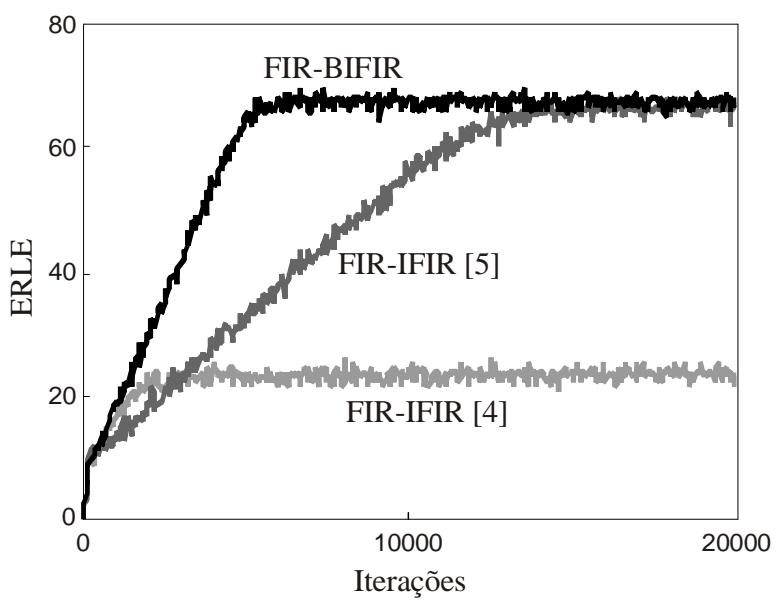

(b)

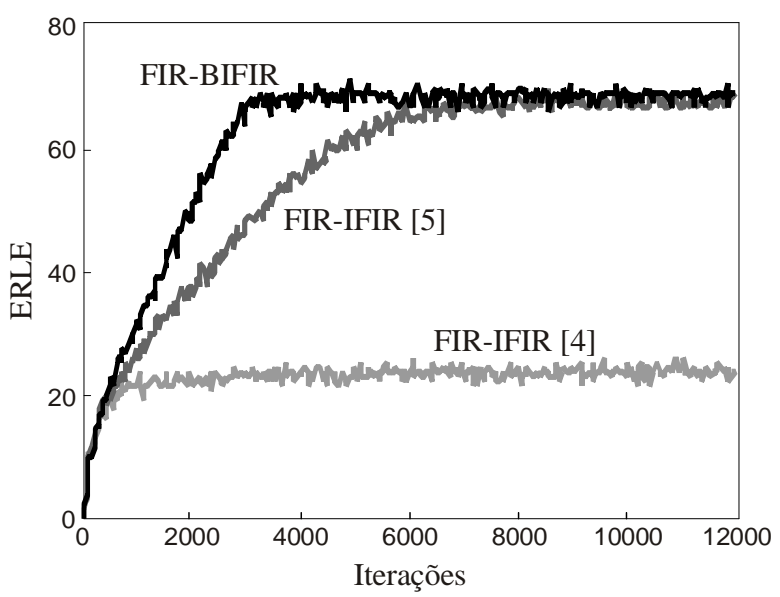

(c)

Fig. 8. Curvas de ERLE (média de 100 realizações). 


\section{CONCLUSÕES}

Neste trabalho, uma nova estratégia para implementação de filtros FIR-IFIR utilizados para o cancelamento de eco em sistemas de comunicação DSL foi apresentada. Em comparação com as outras abordagens FIR-IFIR disponíveis na literatura, o algoritmo proposto apresentou um desempenho superior em termos da ERLE bem como um custo computacional praticamente igual ao da implementação concorrente de menor complexidade. Resultados de simulação foram mostrados corroborando a efetividade da abordagem proposta.

\section{REFERÊNCIAS}

[1] B. Farhang-Boroujeny, Adaptive Filters Theory and Applications. John Wiley \& Sons Ltd., 1999.

[2] E. Hansler and G. Schmidt, Acoustic Echo and Noise Control: A Practical Approach. John Wiley \& Sons Ltd., 2004.

[3] K. Maxwell, “Asymmetric digital subscriber line: interim technology for the nextforty years,” IEEE Commun. Mag., vol. 34, no. 10, pp. 100-106, Oct 1996.

[4] A. Abousaada, T. Aboulnasr, and W. Steenaart, "An echo tail canceller based on adaptive interpolated FIR filtering,” IEEE Trans. Circuits Syst. II, Analog Digit. Signal Process., vol. 39, no. 7, pp. 409-416, Jul. 1992.

[5] S.-S. Lin and W.-R. Wu, "A low-complexity adaptive echo canceller for xDSL Applications,” IEEE Trans. Signal Process., vol. 52, no. 5, pp. 1461-1465, May. 2004.

[6] Y. Neuvo, C. Y. Dong, and S. K. Mitra, "Interpolated finite impulse response digital filters,” IEEE Trans. Acoust., Speech, Signal Process., vol. 32, no. 3, pp. 563-570, Jun. 1984.

[7] E. L. O. Batista, O. J. Tobias, and R. Seara, “A fully adaptive IFIR filter with removed border effect," in Proc. IEEE Int. Conf. Acoust., Speech, Signal Process. (ICASSP), Las Vegas, USA, Apr. 2008, pp. 3821-3824.

[8] E. L. O. Batista, O. J. Tobias, and R. Seara, "Border effect removal for IFIR and interpolated Volterra filters,” in Proc. IEEE Int. Conf. Acoust., Speech, Signal Process. (ICASSP), Honolulu, USA, vol. 3, Apr. 2007, pp. 1329-1332. 